\section{Inhaltliche Koordination 4-2009}

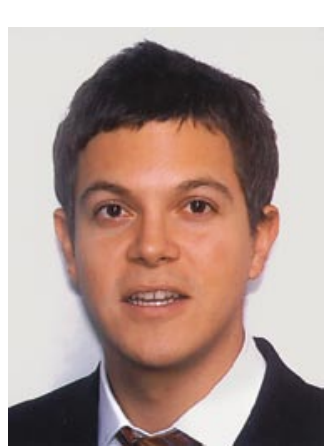

\section{Dipl.-Volkswirt Timo Sohl}

Wissenschaftlicher Mitarbeiter am Forschungszentrum für Handelsmanagement an der Universität St. Gallen

\section{Vorschau 5-2009}

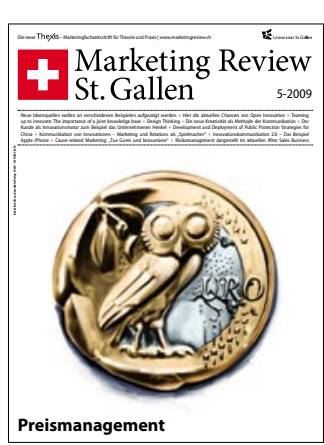

\section{„Preismanagement"}

Die am Markt durchgesetzten Preise bestimmen maßgeblich den Erfolg von Unternehmen. Kein anderes Marketinginstrument hat eine derart hohe Durchschlagskraft und Wirkungsgeschwindigkeit auf den Gewinn. Gleichzeitig kann aber ein nicht systematisches Preismanagement verheerende Konsequenzen für die langfristige Profitabilität haben. Die bedingungslose Teilnahme an Preiskriegen und Rabattschlachten ist hierfür nur ein Beispiel.

Die fünfte Ausgabe der Marketing Review St. Gallen widmet sich genau diesen Herausforderungen. Autoren aus Wissenschaft und Praxis legen die neuesten Erkenntnisse zu den Themen Preisdifferenzierung und -realisierung dar und entwickeln, wissenschaftlich fundiert, Lösungsansätze für ein intelligentes Preismanagement. Darüber hinaus werden unter anderem Aspekte wie Value Based Pricing, Behavioral Pricing oder innovative Ansätze zur Preisbestimmung kritisch beleuchtet.

\title{
Impressum
}

Marketing Review St. Gallen

Die neue Thexis - Fachzeitschrift für Marketing

26. Jahrgang

www.marketingreview.ch

Verlag

Gabler Verlag/GWV Fachverlage GmbH

Abraham-Lincoln-Straße 46

65189 Wiesbaden

Postfach 1546, 65173 Wiesbaden

Geschäftsführer

Dr. Ralf Birkelbach

Albrecht F. Schirmacher

Gesamtleitung Anzeigen: Thomas Werner Gesamtleitung Produktion: Ingo Eichel Gesamtleitung Vertrieb: Gabriel Göttlinger

Verlagsbereichsleitung

Maria Akhavan

\section{Herausgeber}

Prof. Dr. Christian Belz

Prof. Dr. Thomas Bieger

Prof. Dr. Andreas Herrmann

Prof. Dr. Sven Reinecke

Prof. Dr. Thomas Rudolph

Prof. Dr. Marcus Schögel

Prof. Dr. Torsten Tomczak

Redaktionsleitung Universität St. Gallen Dipl.-Kfm. Johannes Hattula

Tel.: +41 (0)71 2242876

johannes.hattula@unisg.ch

Verlagsmitarbeiterin: Karin Halder

Redaktionsleitung Gabler Verlag

Barbara Roscher

Tel.: +49 (0)611 7878-233

barbara.roscher@gabler.de

Redaktion

Barbara Möller

Tel.: +49 (0)611 7878-267

barbara.moeller@gabler.de

Abonnentenbetreuung

VVA-Zeitschriften-Service

Postfach 777, 33310 Gütersloh

Tel.: +49 (0)5241 8019-68

Fax: +49 (0)5241 8096-20

Produktmanagement

Jens Fischer

Tel.: +49 (0)611 7878-340

jens.fischer@gwv-fachverlage.de

Key Account Management

Sabine Schüler

Tel.: +49 (0)611 7878-178

Fax: +49 (0)611 7878-140

sabine.schueler@gwv-media.de

Es gilt die Anzeigenpreisliste Nr. 2

vom 1.1.2009

Anzeigendisposition

Barbara Gerlach,

Tel.: +49 (0)611 7878-198

Fax: +49 (0)611 7878-443

barbara.gerlach@gwv-fachverlage.de

Layout und Produktion

Markus Paulus

Tel.: +49 (0)611 7878-171

markus.paulus@springer.com

Titelbild

Gunter Rubin

rubin@reizend.com
Bezugsmöglichkeit:

Das Heft erscheint sechsmal jährlich.

Bezugspreise Print + Online

(inkl. MwSt. und Versand):

Deutschland: 155,- EUR,

für Studenten 93,- EUR

Schweiz: ca. 263,- CHF

Weltweit: 177,- EUR, für Studenten 103,- EUR

Einzelheftpreis: 34,- EUR zzgl. Versand

Bezugspreise E-Paper/Online only.

(inkl. MwSt)

Weltweit: 133,- EUR, ca. 226,50 CHF

für Studenten 80,- EUR, ca. 136,- CHF

Das Abonnenment kann jederzeit zur nächsten erreichbaren Ausgabe schriftlich mit

Nennung der Kundennummer gekündigt werden. Eine schriftliche Bestätigung erfolgt nicht. Zuviel gezahlte Beträge für nicht gelieferte Ausgaben werden zurückerstattet.

Druck und Verarbeitung

Stürtz, Würzburg

Die Zeitschrift und alle in ihr enthaltenen ein zelnen Beiträge und Abbildungen sind urheberrechtlich geschützt. Jede Verwertung außerhalb der engen Grenzen des Urheberrechtes ist ohne Zustimmung des Verlages unzulässig und strafbar. Das gilt insbesondere für Vervielfältigungen, Übersetzungen, Mikroverfilmungen und die Einspeicherung in elektronischen Systemen. Nachdruckgenehmigung kann die Redaktion erteilen. Für unverlangt eingesandte Beiträge und Rezensionsexemplare wird nicht gehaftet. Jede im Bereich eines gewerblichen Unternehmens hergestellte oder benützte Kopie dient gewerblichen Zwecken gem. §-54-(2)-UrhG und verpflichtet zur Gebührenzahlung an die VG WORT, Abteilung Wissenschaft, Goethestr. 49, 80336 München, von der die einzelnen Zahlungsmodalitäten zu erfragen sind.

Alle Rechte vorbehalten. Kein Teil dieser Zeitschrift darf ohne schriftliche Genehmigung des Verlages vervielfältigt oder verbreitet werden. Unter dieses Verbot fällt insbesondere die gewerbliche Vervielfältigung per Kopie, die Aufnahme in elektronische Datenbanken und die Vervielfältigung auf CD-Rom und allen anderen elektronischen Datenträgern.

Hinweise für Autoren

Der Autor ist mit der Veröffentlichung seines Beitrags damit einverstanden, dass sein Beitrag außer in der Zeitschrift auch durch Lizenzvergabe in anderen Zeitschriften (auch übersetzt), durch Nachdruck in Sammelbänden (z. B. zu Jubiläen der Zeitschrift oder des Verlages oder in Themenbänden), durch längere Auszüge in Büchern des Verlages auch zu Werbezwecken, durch Vervielfältigung und Verbreitung auf CDROM oder anderen Datenträgern, durch Speicherung auf Datenbanken, deren Weitergabe und den Abruf von solchen Datenbanken während der Dauer des Urheberrechtsschutzes an dem Beitrag im In- und Ausland vom Verlag und seinen Lizenznehmern genutzt wird.

(c) Gabler Verlag/GWV Fachverlage GmbH, Wiesbaden 2009.

Der Verlag ist ein Unternehmen von Springe Science+Business Media. ISSN 1865-6544 